\title{
MHD Free Convection from a Semi-infinite Vertical Porous Plate with Diffusion-Thermo Effect
}

\author{
Rajdeep Bordoloi 1,*(D), Nazibuddin Ahmed ${ }^{2}$ (D) \\ 1 Department of Mathematics, Gauhati University, Assam, India; rajdeepbordoloi24@gmail.com (R.B.); \\ nazib@gauhati.ac.in (N.A.); \\ * Correspondence: rajdeepbordoloi24@gmail.com (R.B.);
}

Scopus Author ID 9636158600

Received: 13.09.2021; Revised: 4.11.2021; Accepted: 6.11.2021; Published: 28.11.2021

\begin{abstract}
An analytical solution for two-dimensional unsteady MHD free convective mass transfer flows of viscous incompressible optically thin fluid past a semi-infinite vertical porous plate in the presence of thermal radiation and chemical reaction is presented in this paper. A uniform magnetic field is applied normally to the plate with a first-order chemical reaction. The non-dimensional governing equations are solved analytically by using the regular perturbation technique. The effects of various physical parameters like radiation parameter $Q$, Dufour effect $D u$, chemical reaction parameter $K$, thermal Grashof number $G r$, Hartmann number $M$, porosity parameter $k$, etc., are studied and demonstrated graphically. One of the significant findings of this analysis includes that an intensification of the chemical reaction effect causes a downfall in the fluid concentration. In contrast, another important outcome of the present study is that the rate of heat transfer and shear stress at the wall increases under the diffusion thermo effect or Dufour effect. Still, it tends to fall for high radiation. Further, the rate of mass transfer rises under the chemical reaction effect.
\end{abstract}

Keywords: MHD; Dufour effect; thermal radiation; chemical reaction; porosity.

(C) 2021 by the authors. This article is an open-access article distributed under the terms and conditions of the Creative Commons Attribution (CC BY) license (https://creativecommons.org/licenses/by/4.0/).

\section{Nomenclature:}

C Species concentration of the fluid; K.mol. $\mathrm{m}^{-3}$

$\bar{C}_{\infty}$ Species concentration of the fluid far away from the plate; K.mol.m ${ }^{-3}$

$\bar{C}_{w}$ Species concentration of the fluid near the plate; K.mol

$C_{p}$ Specific heat at constant pressure; $J . \mathrm{kg}^{-1} \cdot \mathrm{K}^{-1}$

$D_{M}$ Chemical molecular diffusivity; $m^{2} \cdot s^{-1}$

$\rho$ Density of the fluid; $\mathrm{kg} \cdot \mathrm{m}^{-3}$

$g$ Acceleration due to gravity; $m . s^{-2}$

$q_{r}$ Radiative heat flux; $W . m^{-2}$

$T$ Temperature of the fluid; $K$ $\bar{u}$ Component of velocity along $x$-axis; $m \cdot s^{-1}$

$\bar{v}$ Component of velocity along y-axis; $m \cdot s^{-1}$

$v$ Velocity of the fluid; $m . s^{-1}$

$v_{0}$ Scale of the suction velocity; $m . s^{-1}$

Greek symbols

$\mu$ Coefficient of viscosity; $\mathrm{kg} \cdot \mathrm{m}^{-1} \cdot \mathrm{s}^{-1}$

$\kappa$ Thermal conductivity; $W \cdot m^{-1} \cdot K^{-1}$

$\sigma$ Electrical conductivity; $\frac{1}{(\mathrm{Ohm} \times m)}$

$v$ Kinematic viscosity; $\mathrm{m}^{2} . \mathrm{s}^{-1}$ 
$\bar{T}_{w}$ Temperature of the plate; $K$

$\bar{t}$ Time

\section{Introduction}

For several decades, magnetohydrodynamics (MHD) has attracted numerous scientists and engineers because of its fascination and importance in various technological devices and in understanding diverse cosmic phenomena. MHD concepts are used in designing heat exchangers, pumps, flow meters, and power generation systems, and confinement schemes for controlled fusion. MHD convection problems are extremely important in the study of aeronautics, particularly in missile aerodynamics, because the temperatures that occur at such high speeds are sufficient to dissociate or even ionize the air significantly. MHD flow has applications in metrology, solar physics, and the motion of the 'earth's core. The concepts of MHD are widely used in medicine and biology. Problems involving convection with hydromagnetic fluxes are also important in geophysics, astrophysics, plasma physics, missile technology, and other fields. The current form of MHD is based on the pioneering contributions of many authors Alfven [1], Cowling [2], Shercliff [3], Ferraro and Plumpton [4], and Crammer and Pai [5]. Several authors have contributed to MHD in recent times; some of them are Raghunath et al. [6], Anwar et al. [7], Gaud et al. [8], Sheri et al. [9], Omamoke et al. [10], Seth et al. [11], etc.

Natural convection is a heat transfer phenomenon in which the driver of the fluid motion is self-induced forces. These forces may be due to temperature or concentration gradients. It has many applications in chemical engineering, petroleum technology, agricultural engineering, etc. Recently, Ahmed and Choudhury [12], Sinha and Ahmed [13], Allan and Dardery [14], Chamuah et al. [15], Ahmed and Dutta [16], Mahato et al. [17], and many authors have studied MHD free convective heat and mass transfer flow through a porous medium.

The phenomenon by which heat transfer is caused by concentration gradient is called diffusion-thermo or Dufour effect. The concentration gradient results in a temperature change. The effect was first observed by the Swiss physicist L. Dufour in 1873. It has enormous applications in many fields, especially in CVD problems and chemical reactors. Recently, Dagana and Amos [18] investigated MHD free convection heat and mass transfer flow in a porous medium with Dufour and chemical reaction effects. Reddy [19] studied Dufour's effects on heat and mass transfer of non-Newtonian power-law fluid over a porous plate. Ahmed et al. [20] examined three-dimensional hydromagnetic convective flow past a porous vertical plate with sinusoidal suction in slip flow regime, taking the Soret and Dufour effect into account. Jha and Sarki [21] worked on Chemical reaction and Dufour effects on nonlinear free convection heat and mass transfer flow near a vertical moving porous plate. Dufour's effect on MHD free convection heat and mass transfer flow over an inclined plate embedded in a porous medium was investigated by Islam et al. [22].

In recent times, many researchers have given considerable interest to the study of MHD in the presence of radiation. Mishra et al. [23] studied the effect of radiation and non-uniform heat source on the unstable, MHD viscous fluid through a heated plate with time-dependent suction and viscous dissipation. Chiranjeevi et al. [24] investigated the problem of the MHD boundary layer flow analysis in the presence of thermal absorption with heat generation and chemical reaction over a plate. To simulate the 2D unstable magneto-hydrodynamic flow of an 
electrically conducting fluid over a permeable plate under the effect of thermal radiation and chemical reaction, a mathematical model has been developed by Suneetha et al. [25]. Gaud [26] examined the impacts of thermal radiation on the MHD stagnation point stream over a stretching sheet with slip boundary conditions.

In chemical engineering, chemical reactions have always played an important role in heat and mass transfer. A theoretical analysis has been done by Rajput and Kumar [27] to study the unsteady MHD flow through porous medium past an exponentially accelerated vertical plate with variable wall temperature and mass diffusion in the presence of Hall current, radiation, and chemical reaction. Thermal radiation on unsteady electrical MHD flow of nanofluid overstretching sheet with chemical reaction was investigated by Daniel et al. [28]. Reddy et al. [29] investigated the effects of chemical reaction on unsteady MHD flow past an impulsively started oscillating infinite vertical plate with variable temperature and constant mass diffusion in the presence of Hall current. Suneetha et al. [30] studied the combined effects of thermal radiation and chemical reaction on steady MHD mixed convective heat and mass transfer flow past a vertical surface under the influence of Joule and viscous dissipation. Rajakumar et al. [31] studied radiation, dissipation, and Dufour effects on MHD free convection Casson fluid flow through a vertical oscillatory porous plate with the ion-slip current. Obulesu et al. [32] discussed current hall effects on MHD convective flow past a porous plate with thermal radiation, chemical reaction with radiation absorption. Recently, an analytical solution based on the asymptotic series expansion method is presented by Ahmed and Bordoloi [33] for the problem of a fully developed chemically reactive laminar threedimensional flow through a porous vertical infinite plate in a porous medium sinusoidal permeability as suction in the existence of appreciable radiation. Seth et al. [34] investigated unsteady MHD flow of a Casson fluid near a vertical oscillating plate through a non-Darcy porous medium. Kumar et al. [35] explained the effect of magnetite nanofluid, taking into account water as the base fluid, over a rotating disk in the presence of the external magnetic field.

Because of the above studies, this paper investigates the effect of thermal radiation and chemical reaction on MHD free convective mass transfer flow of viscous incompressible optically thin fluid with Dufour effect using Cogley et al. [36] model. The novelty of the present investigation is that the present problem deals with a flow through a porous medium of Brickman type in the presence of a transverse magnetic field. Rosseland type of radiation, diffusion-thermo effect, and homogenous first-order chemical reaction are also considered in our study's preview. The asymptotic series expansion method is utilized for getting the solution of the governing equations. It is worthwhile to mention that asymptotic series expansion is unconditionally convergent. The Present investigation may impact various engineering processes such as paper production, glass blowing, extrusion of plastic sheets, etc.

\section{Materials and Methods}

\subsection{Basic equations.}

Equation of continuity:

$$
\vec{\nabla} \cdot \vec{q}=0
$$

Momentum equation: 


$$
\rho\left[\frac{\partial \vec{q}}{\partial \bar{t}}+(\vec{q} \cdot \vec{\nabla}) \vec{q}\right]=\vec{F}-\vec{\nabla} \bar{p}+\mu \nabla^{2} \vec{q}+\vec{J} \times \vec{B}-\mu \frac{\vec{q}}{\vec{k}}
$$

Energy equation:

$$
\rho C_{p}\left[\frac{\partial \bar{T}}{\partial \bar{t}}+(\vec{q} \cdot \vec{\nabla}) \bar{T}\right]=\kappa \nabla^{2} \bar{T}-\vec{\nabla} \cdot q_{r}+\frac{\rho D_{M} K_{T}}{C_{S}} \nabla^{2} \bar{C}
$$

Species continuity equation:

$$
\frac{\partial \bar{C}}{\partial \bar{t}}+(\vec{q} \cdot \vec{\nabla}) \bar{C}=D_{M} \nabla^{2} \bar{C}+K^{*}\left(\bar{C}_{\infty}-\bar{C}\right)
$$

\subsection{Mathematical formulation.}

A cartesian coordinate system $(\bar{x}, \bar{y}, \bar{z})$ with the $\mathrm{X}$-axis along with the infinite vertical plate, the Y-axis normal to the plate, and the Z-axis alongside the width of the plate has been considered. Initially, the plate and the fluid were at the same temperature $\bar{T}_{\infty}$ with concentration levels $\bar{C}_{\infty}$ at all points. At the time, $\bar{t}>0$, the plate temperature was suddenly raised $\bar{T}_{w}$, and the concentration level at the plate rose $\bar{C}_{w}$. A uniform magnetic field is applied normally to the plate.

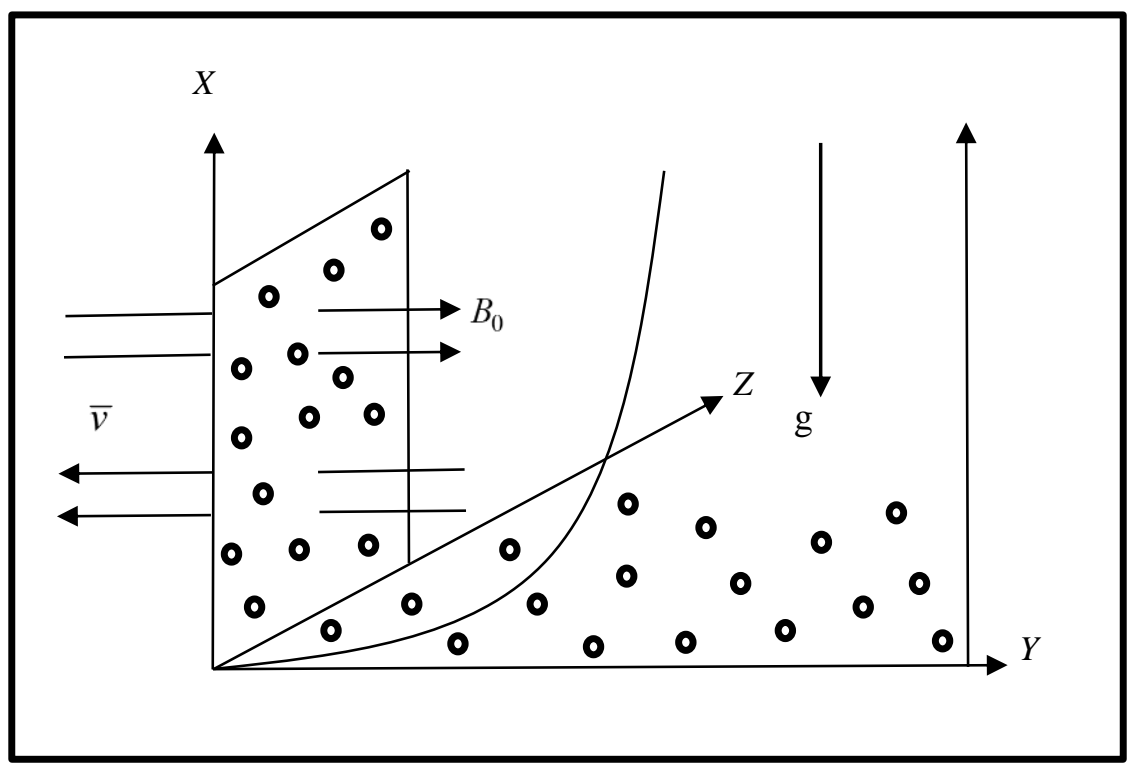

Figure 1. Physical configuration.

To make the mathematical model of the theoretical problem idealized, the following constraints are imposed:

(a) The fluid is incompressible, viscous electrically conducting.

(b) All the fluid properties are constant, excluding density in the buoyancy force term.

(c) External electric field is negligible.

(d) Ohmic dissipation, as well as viscous dissipation, is not considered.

(e) Induced magnetic field in comparison to the applied magnetic field is negligible.

(f) No external electric field is applied, for which $\vec{E}=0$

Gauss's law of magnetism

$$
\operatorname{Div} \vec{B}=0
$$


Electric current density

$$
\vec{J}=\sigma[\vec{E}+\vec{q} \times \vec{B}]
$$

$\vec{B}=B_{0} \hat{j}$, is the uniform magnetic field with strength $B_{0}$ applied normal to the plate, directed into the fluid region. Due to semi-infinite plate surface assumptions, all the flow variables except pressure are functions of $\bar{y}$ and $\bar{t}$ only.

Thus, the governing equations take the forms as given below:

A. Continuity equation

$$
\frac{\partial \bar{v}}{\partial \bar{y}}=0
$$

It is seen from equation (7) that $\bar{v}$ it is a constant. Assuming the suction velocity to be oscillatory, we have

$$
\bar{v}=-v_{0}\left(1+\varepsilon A e^{i \omega t}\right)
$$

Where $\varepsilon, A$ are small such that $\varepsilon A<<1$. The negative sign indicates that the suction velocity is directed towards the plate.

$\mathrm{X}$ - component of momentum equation

$$
\rho\left[\frac{\partial \bar{u}}{\partial \bar{t}}+\left(\bar{v} \frac{\partial}{\partial \bar{y}}\right) \bar{u}\right]=-\rho g-\frac{\partial \bar{p}}{\partial \bar{x}}+\mu \frac{\partial^{2} \bar{u}}{\partial \bar{y}^{2}}-\sigma B_{0}^{2} \bar{u}-\frac{\mu \bar{u}}{\bar{k}}
$$

In the free stream, equation (8) takes the form

$$
0=-\rho_{\infty} g-\frac{\partial \bar{p}}{\partial \bar{x}}
$$

Subtracting (10) from (9), we obtain

$$
\rho\left[\frac{\partial \bar{u}}{\partial \bar{t}}+\bar{v} \frac{\partial \bar{u}}{\partial \bar{y}}\right]=\left(\rho_{\infty}-\rho\right) g+\mu \frac{\partial^{2} \bar{u}}{\partial \bar{y}^{2}}-\sigma B_{0}^{2} \bar{u}-\frac{\mu \bar{u}}{\bar{k}}
$$

Volume expression gives

$$
\rho_{\infty}-\rho=\rho\left[\beta\left(\bar{T}-\bar{T}_{\infty}\right)+\beta^{*}\left(\bar{C}-\bar{C}_{\infty}\right)\right]
$$

Using (12) in (11), we get

B. $\bar{x}$ - component of momentum equation [12],

$$
\frac{\partial \bar{u}}{\partial \bar{t}}+\bar{v} \frac{\partial \bar{u}}{\partial \bar{y}}=g \beta\left(\bar{T}-\bar{T}_{\infty}\right)+g \beta^{*}\left(\bar{C}-\bar{C}_{\infty}\right)+v \frac{\partial^{2} \bar{u}}{\partial \bar{y}^{2}}-\frac{\sigma B_{0}^{2} \bar{u}}{\rho}-\frac{v \bar{u}}{\bar{k}}
$$

Now the conservation of energy equation [21],

$$
\frac{\partial \bar{T}}{\partial \bar{t}}+\bar{v} \frac{\partial \bar{T}}{\partial \bar{y}}=\frac{\kappa}{\rho C_{p}} \frac{\partial^{2} \bar{T}}{\partial \bar{y}^{2}}-\frac{1}{\rho C_{p}} \frac{\partial q_{r}}{\partial \bar{y}}+\frac{D_{M} K_{T}}{C_{p} C_{S}} \frac{\partial^{2} \bar{C}}{\partial \bar{y}^{2}}
$$

According to Cogley's model, the radiative heat flux in optically thin non-Gray gas near equilibrium is specified by

$$
\frac{\partial q_{r}}{\partial \bar{y}}=4 I\left(\bar{T}-\bar{T}_{\infty}\right), \text { where } I=\int_{0}^{\infty}\left(k_{\lambda}\right)_{w}\left(\frac{\partial e_{\lambda b}}{\partial \bar{T}}\right)_{w} d \lambda
$$

Unification of (15) and (14) leads

C. Energy equation $[12,21]$,

$$
\frac{\partial \bar{T}}{\partial \bar{t}}+\bar{v} \frac{\partial \bar{T}}{\partial \bar{y}}=\frac{\kappa}{\rho C_{p}} \frac{\partial^{2} \bar{T}}{\partial \bar{y}^{2}}-\frac{1}{\rho C_{p}} 4 I\left(\bar{T}-\bar{T}_{\infty}\right)+\frac{D_{M} K_{T}}{C_{p} C_{S}} \frac{\partial^{2} \bar{C}}{\partial \bar{y}^{2}}
$$


D. Mass transfer equation [10],

$$
\frac{\partial \bar{C}}{\partial \bar{t}}+\bar{v} \frac{\partial \bar{C}}{\partial \bar{y}}=D_{M} \frac{\partial^{2} \bar{C}}{\partial \bar{y}^{2}}+K^{*}\left(\bar{C}_{\infty}-\bar{C}\right)
$$

The corresponding boundary conditions are

$$
\left.\begin{array}{l}
t>0, \bar{u}=0, \bar{T}=\bar{T}_{\infty}+\left(\bar{T}_{w}-\bar{T}_{\infty}\right)\left(1+\varepsilon e^{i \bar{\omega} \bar{t}}\right), \bar{C}=\bar{C}_{w} \text { at } \bar{y}=0 \\
\bar{u} \rightarrow 0, \bar{T} \rightarrow \bar{T}_{\infty}, \bar{C} \rightarrow \bar{C}_{\infty} \text { as } \bar{y} \rightarrow \infty
\end{array}\right\}
$$

To make the mathematical model normalized, we introduce the following nondimensional quantities:

$$
\begin{aligned}
& \left.\begin{array}{l}
y=\frac{v_{0} \bar{y}}{v}, u=\frac{\bar{u}}{v_{0}}, t=\frac{\bar{t} v_{0}^{2}}{4 v}, \theta=\frac{\bar{T}-\bar{T}_{\infty}}{\bar{T}_{w}-\bar{T}_{\infty}}, \phi=\frac{\bar{C}-\bar{C}_{\infty}}{\bar{C}_{w}-\bar{C}_{\infty}}, S c=\frac{v}{D_{M}}, v=\frac{\bar{v}}{v_{0}}, \\
G r=\frac{g \beta v\left(\bar{T}_{w}-\bar{T}_{\infty}\right)}{v_{0}^{3}}, G c=\frac{g \beta^{*} v\left(\bar{C}_{w}-\bar{C}_{\infty}\right)}{v_{0}^{3}}, \omega=\frac{4 v \bar{\omega}}{v_{0}^{2}}, M=\frac{\sigma B_{0}^{2} v}{\rho v_{0}^{2}}, \\
k=\frac{\bar{k} v_{0}^{2}}{v^{2}}, K=\frac{K^{*} v}{v_{0}^{2}}, D u=\frac{D_{M} K_{T}\left(\bar{C}_{w}-\bar{C}_{\infty}\right)}{v C_{p} C_{S}\left(\bar{T}_{w}-\bar{T}_{\infty}\right)}, \operatorname{Pr}=\frac{\mu C_{p}}{\kappa}, Q=\frac{4 I v}{\rho C_{p} v_{0}^{2}}
\end{array}\right\}
\end{aligned}
$$

Under the transformations (19), the governing equations (13), (16), (17) reduces to the following forms

$$
\begin{gathered}
\frac{1}{4}\left(\frac{\partial u}{\partial t}\right)-\left(1+\varepsilon A e^{i \omega t}\right) \frac{\partial u}{\partial y}=G r \theta+G c \phi+\frac{\partial^{2} u}{\partial y^{2}}-\left(M+\frac{1}{k}\right) u \\
\frac{1}{4}\left(\frac{\partial \phi}{\partial t}\right)-\left(1+\varepsilon A e^{i \omega t}\right) \frac{\partial \phi}{\partial y}=\frac{1}{S c} \frac{\partial^{2} \phi}{\partial y^{2}}-K \phi \\
\frac{1}{4}\left(\frac{\partial \theta}{\partial t}\right)-\left(1+\varepsilon A e^{i \omega t}\right) \frac{\partial \theta}{\partial y}=\frac{1}{\operatorname{Pr}} \frac{\partial^{2} \theta}{\partial y^{2}}-Q \theta+D u \frac{\partial^{2} \phi}{\partial y^{2}}
\end{gathered}
$$

With conditions:

$$
\left.\begin{array}{l}
t>0, u=0, \theta=\theta_{w}=1+\varepsilon A e^{i \omega t}, \phi=1 \text { at } y=0 \\
u \rightarrow 0, \theta \rightarrow 0, \phi \rightarrow 0 \text { as } y \rightarrow \infty
\end{array}\right\}
$$

\subsection{Method of solutions.}

To solve the equations (20), (21), and (22) under the conditions (23), we consider the following asymptotic form

$$
\left.\begin{array}{l}
u(y, t)=u_{0}(y)+\varepsilon e^{i \omega t} u_{1}(y)+O\left(\varepsilon^{2}\right) \\
\theta(y, t)=T_{0}(y)+\varepsilon e^{i \omega t} T_{1}(y)+O\left(\varepsilon^{2}\right) \\
\phi(y, t)=C_{0}(y)+\varepsilon e^{i \omega t} C_{1}(y)+O\left(\varepsilon^{2}\right)
\end{array}\right\}
$$

Substituting (24) in the equations (20), (21), (22) under the condition (23) and equating the harmonic and non-harmonic terms, and also neglecting $O\left(\varepsilon^{2}\right)$, following differential equations are obtained.

$$
\begin{gathered}
u_{0}^{\prime \prime}+u_{0}^{\prime}-\left(M+\frac{1}{k}\right) u_{0}=-G r \theta_{0}-G c \phi_{0} \\
u_{1}^{\prime \prime}+u_{1}^{\prime}-\left(M+\frac{1}{k}+\frac{i \omega}{4}\right) u_{1}=-A u_{0}^{\prime}-G r \theta_{1}-G c \phi_{1}
\end{gathered}
$$




$$
\begin{gathered}
\theta_{0}^{\prime \prime}+\operatorname{Pr} \theta_{0}^{\prime}-\operatorname{Pr} Q \theta_{0}=-\operatorname{Pr} D u \phi_{0}^{\prime \prime} \\
\theta_{1}^{\prime \prime}+\operatorname{Pr} \theta_{1}^{\prime}-\left(Q+\frac{i \omega}{4}\right) \operatorname{Pr} \theta_{1}=-A \operatorname{Pr} \theta_{0}^{\prime}-\operatorname{Pr} D u \phi_{1}^{\prime \prime} \\
\phi_{0}^{\prime \prime}+S c \phi_{0}^{\prime}-S c K \phi_{0}=0 \\
\phi_{1}^{\prime \prime}+S c \phi_{1}^{\prime}-\left(K+\frac{i \omega}{4}\right) S c \phi_{1}=-S c A \phi_{0}^{\prime}
\end{gathered}
$$

Subject to the conditions:

$$
\left.\begin{array}{l}
t>0, u_{0}=u_{1}=0, \theta_{0}=\theta_{1}=1, \phi_{0}=1, \phi_{1}=0, \text { at } \mathrm{y}=0 \\
u_{0}=u_{1} \rightarrow 0, \theta_{0}=\theta_{1} \rightarrow 0, \phi_{0}=\phi_{1} \rightarrow 0 \text { as } y \rightarrow \infty
\end{array}\right\}
$$

Solving equations (25) to (30) by using perturbation technique and using the boundary conditions (31), the solution for velocity, temperature, and concentration, respectively, are expressed as

$$
\begin{aligned}
& u=\left(A_{6}-A_{7}\right) e^{-m_{5} y}-A_{6} e^{-m_{3} y}+A_{7} e^{-m_{1} y}+\varepsilon e^{i \omega t}\left[\left(-A_{8}-A_{9}-A_{10}+A_{11}-A_{12}\right) e^{-m_{6} y}\right. \\
& \left.+A_{8} e^{-m_{5} y}+A_{9} e^{-m_{3} y}+A_{10} e^{-m_{1} y}-A_{11} e^{-m_{4} y}+A_{12} e^{-m_{2} y}\right] \\
& \theta=\left(1+A_{2}\right) e^{-m_{3} y}-A_{2} e^{-m_{1} y}+\varepsilon e^{i \omega t}\left[\left(1+A_{3}+A_{4}-A_{5}\right) e^{-m_{4} y}-A_{3} e^{-m_{3} y}-A_{4} e^{-m_{1} y}+A_{5} e^{-m_{2} y}\right] \\
& \phi=e^{-m_{1} y}+A_{1} \varepsilon e^{i \omega t}\left(e^{-m_{1} y}-e^{-m_{2} y}\right)
\end{aligned}
$$

The non-dimensional skin friction $\tau$ at the plate $y=0$ is given by

$$
\begin{aligned}
& \tau=\left.\frac{\partial u}{\partial y}\right]_{y=0} \\
&=-m_{5}\left(A_{6}-A_{7}\right)+m_{3} A_{6}-m_{1} A_{7}+\varepsilon e^{i \omega t}\left[-\left(-A_{8}-A_{9}-A_{10}+A_{11}-A_{12}\right) m_{6}\right. \\
&\left.\quad-m_{5} A_{8}-m_{3} A_{9}-m_{1} A_{10}+m_{4} A_{11}-m_{2} A_{12}\right]
\end{aligned}
$$

The rate of heat transfer at the plate in terms of the Nusselt number based on Fourier's law of heat conduction is expressed as

$$
\begin{aligned}
N u & \left.=\frac{\partial \theta}{\partial y}\right]_{y=0} \\
& =-\left(1+A_{2}\right) m_{3}+A_{2} m_{1}+\varepsilon e^{i \omega t}\left[-\left(1+A_{3}+A_{4}-A_{5}\right) m_{4}+A_{3} m_{3}+A_{4} m_{1}-A_{5} m_{2}\right]
\end{aligned}
$$

The rate of concentration in terms of Sherwood number is given by the relation

$$
\begin{aligned}
S h & \left.=\frac{\partial \phi}{\partial y}\right]_{y=0} \\
& =-m_{1}+A_{1} \varepsilon e^{i \omega t}\left(m_{2}-m_{1}\right)
\end{aligned}
$$

\section{Results and Discussion}

To get the physical insight into the problem, the effects of various parameters like radiation parameter $Q$, Dufour effect $D u$, Schmidt number $S c$, thermal Grashof number $G r$, Hartmann number $M$, porosity parameter $k$, etc. are studied graphically on velocity, temperature, concentration, skin friction, Nusselt number and Sherwood number which are portrayed from Figures 2-14. The default values for emerging flow parameters are chosen as 
$\operatorname{Pr}=0.71, \mathrm{Sc}=0.7, \mathrm{Gr}=5, \mathrm{Gc}=5, k=15, K=5, D u=10, Q=5, M=10, \varepsilon=0.01, A=0.01, \omega=2$, $t=0.2$ until otherwise specified particularly.

The effects of $D u, G r$, and $M$ on velocity profiles are displayed in Figures 2, 3, and 4, respectively. Figures 2 and 3 shows that fluid velocity increases with the increasing values of $D u$ and $G r$. Thus, the velocity of the fluid gets accelerated under diffusion thermo effect and thermal buoyancy force. From Figure 4, we see that the transverse magnetic field $M$ tends to retard the fluid motion. This is because of the Lorentz force, which decelerates the fluid motion.

The variations in temperature versus normal coordinate y are demonstrated in figures 5 to 7 . Figure 5 predicts a clear rise in the fluid temperature under diffusion thermal effect $D u$. Also, it is observed from Figure 6 that a rise in the positive values of radiation parameter $Q$ results in a significant downfall of the magnitude of the temperature. Figure 7 shows that fluid temperature increases under chemical reaction parameters in a thin layer near the plate, and thereafter this behavior takes a reverse turn.

The effect of chemical reaction parameter $K$ and Schmidt number $S c$ on the concentration is shown in Figures 8 and 9, respectively. It is seen that the concentration of the species decreases substantially due to the rise in chemical reaction effect and Schmidt number.

It is observed in Figure 10 that viscous drag gets accelerated for increasing the Dufour number $D u$. Thus, the diffusion-thermo effect has a considerable contribution in enhancing the frictional resistance on the plate. Whereas, reverse nature is seen for radiation $Q$ in Figure 11. Figure 11 shows that viscous drag at the plate decreases as the radiation increases.

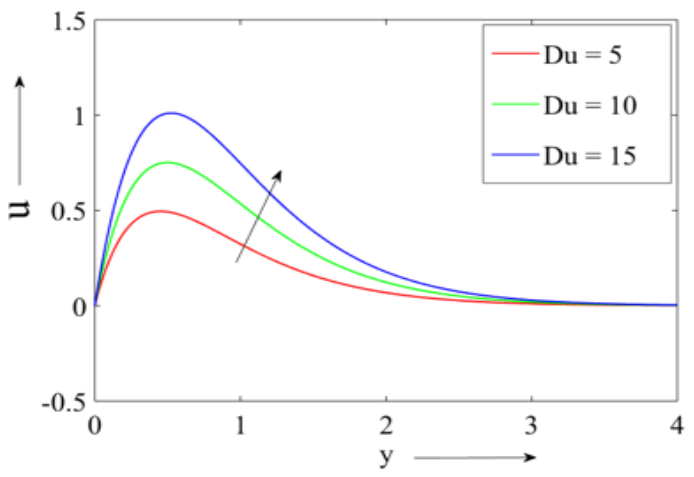

Figure 2. Velocity u vs y for variations in $D u$ when $\operatorname{Pr}=0.71, S c=0.7, k=15, K=5, Q=5, G r=5, G c=5, M=10$, $\varepsilon=0.01, A=0.01, \omega=2, t=0.2$.

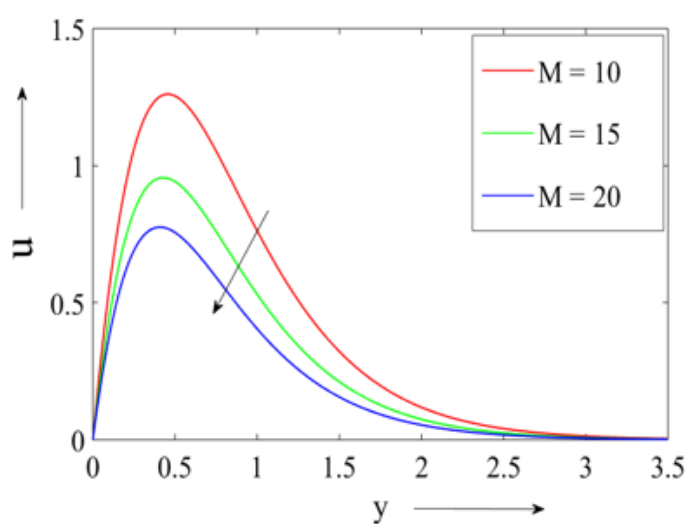

Figure 4. Velocity u vs y for variations in $M$ when $\operatorname{Pr}=0.71, S c=0.7, k=15, K=20, Q=5, D u=10, G r=5$, $G c=5, \varepsilon=0.01, A=0.01, \omega=2, t=0.2$.

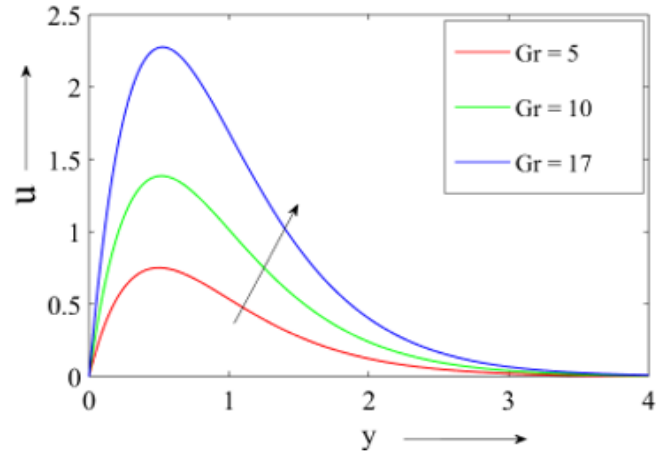

Figure 3. Velocity u vs y for variations in $G r$ when $\operatorname{Pr}=0.71, S c=0.7, k=15, K=5, Q=5, D u=10, G c=5$, $M=10, \varepsilon=0.01, A=0.01, \omega=2, t=0.2$.

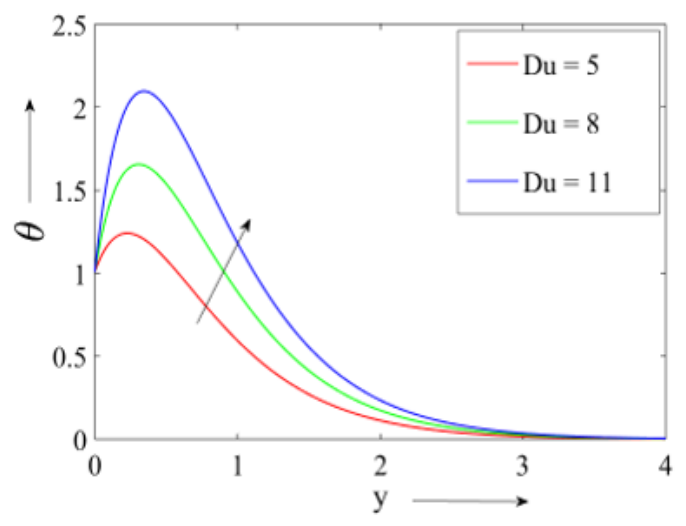

Figure 5. Temperature $\theta$ vs y for variations in $D u$ when $\operatorname{Pr}=0.71, S c=0.7, K=5, Q=5, \varepsilon=0.01$, $A=0.01, \omega=2, t=0.2$. 


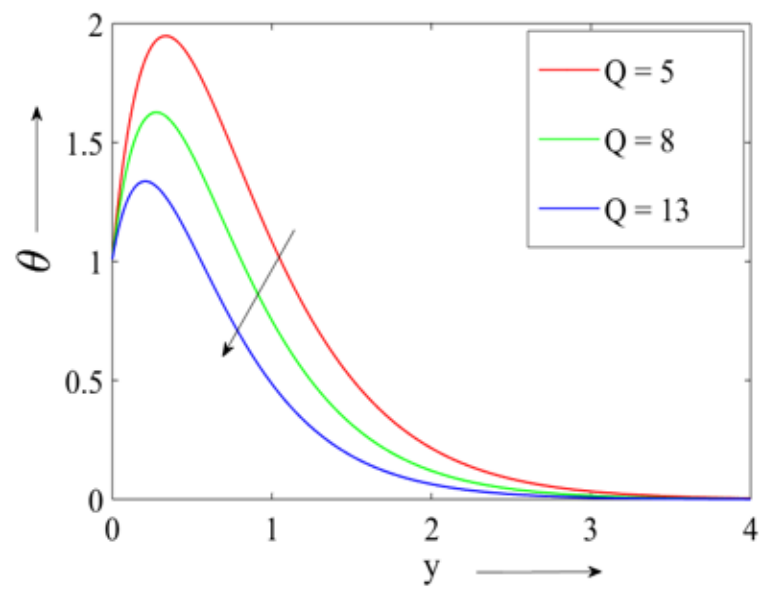

Figure 6. Temperature $\theta$ vs y for variations in $Q$ when $\operatorname{Pr}=0.71, S c=0.7, K=5, D u=10, \varepsilon=0.01, A=0.01$, $\omega=2, t=0.2$.

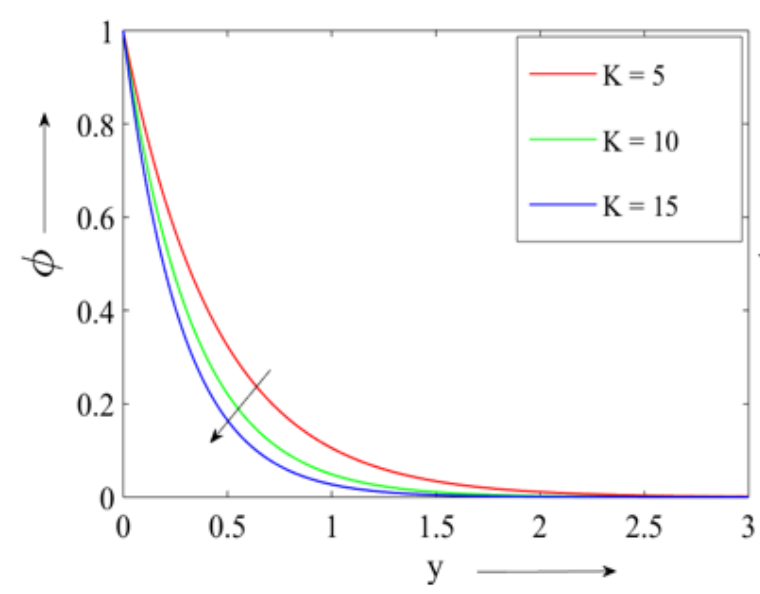

Figure 8. Concentration $\phi$ vs y for variations in $K$ when $S c=0.7, \varepsilon=0.01, A=0.01, \omega=2, t=0.2$.

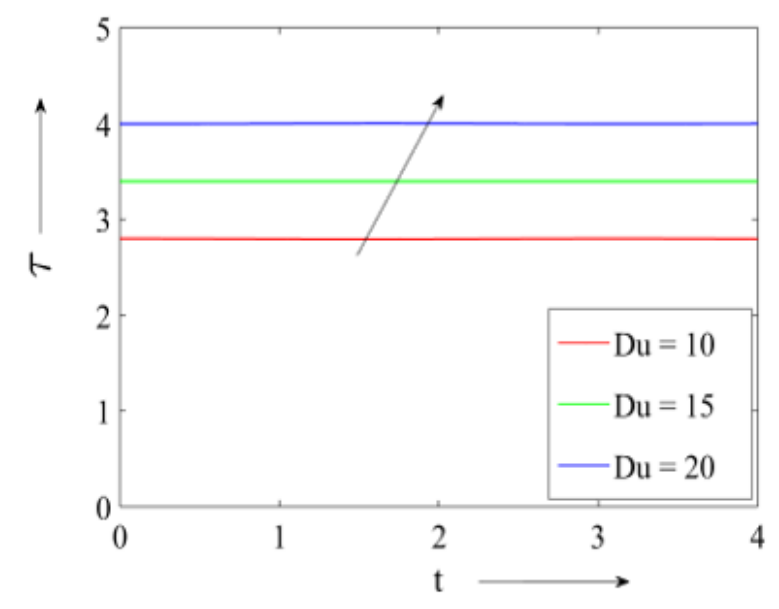

Figure 10. Skin friction $\tau$ vs $t$ for variations in $D u$ when $\operatorname{Pr}=0.71, S c=0.7, k=15, K=5, Q=5, G r=5, G c=5$, $M=10, \varepsilon=0.01, A=0.01, \omega=2$.

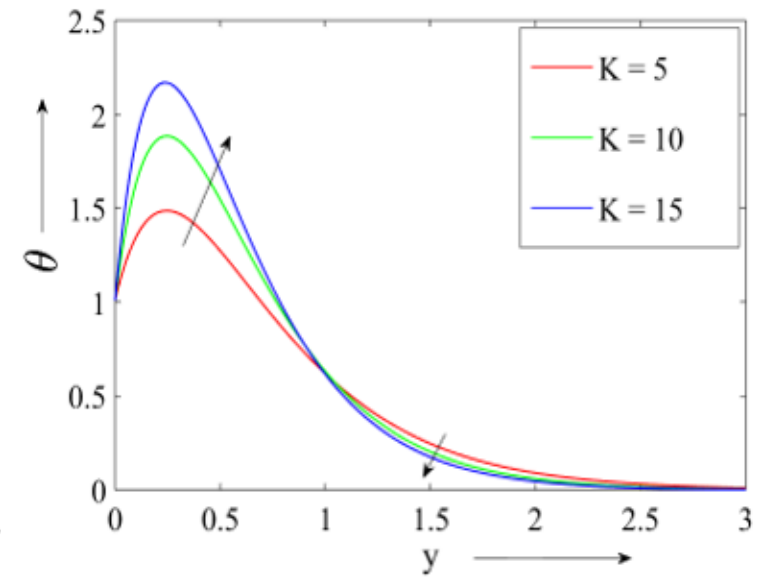

Figure 7. Temperature $\theta$ vs y for variations in $K$ when $\operatorname{Pr}=0.71, S c=0.7, Q=5, D u=10, \varepsilon=0.01$, $A=0.01, \omega=2, t=0.2$.

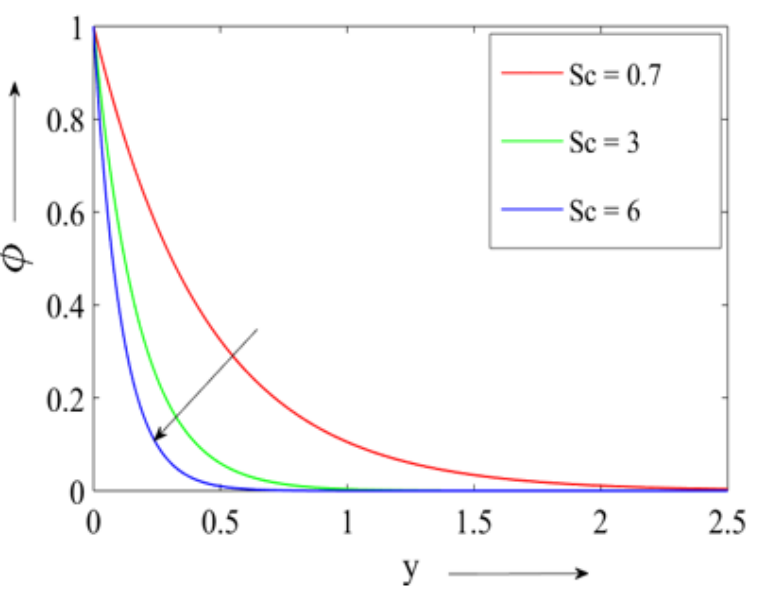

Figure 9. Concentration $\phi$ vs y for variations in $S c$ when $K=5, \varepsilon=0.01, A=0.01, \omega=2, t=0.2$.

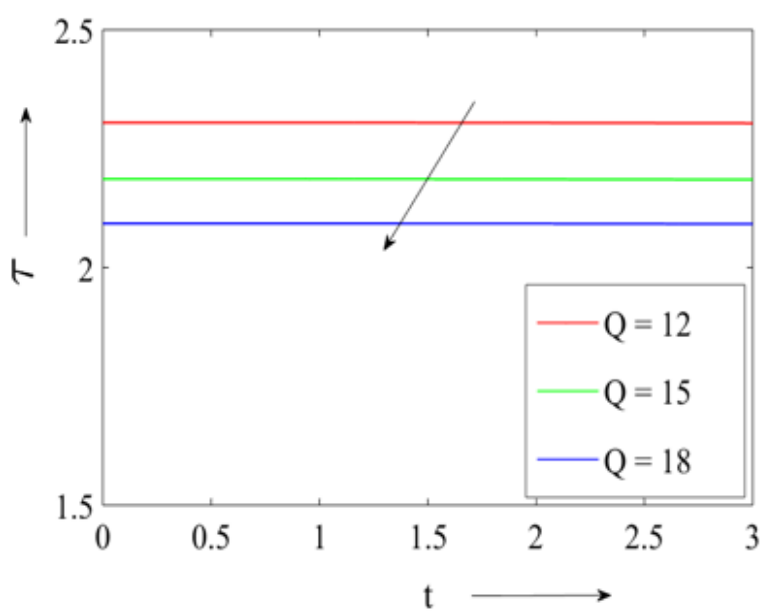

Figure 11. Skin friction $\tau$ vs $t$ for variations in $Q$ when $\operatorname{Pr}=0.71, S c=0.7, k=15, K=5, D u=10, G r=5$, $G c=5, M=10, \varepsilon=0.01, A=0.01, \omega=2$. 


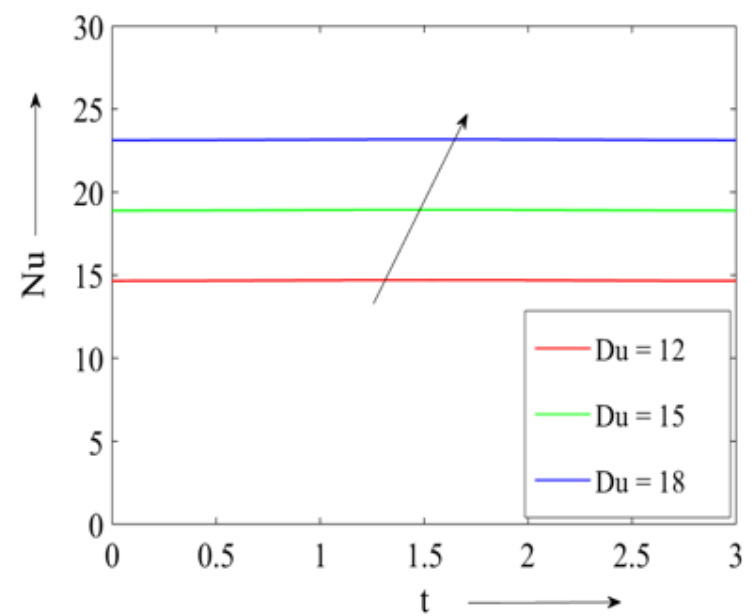

Figure 12. Nusselt number $N u$ vs $t$ for variations in $D u$ when $\operatorname{Pr}=0.71, S c=0.7, K=5, Q=5, \varepsilon=0.01, A=0.01$, $\omega=2$.

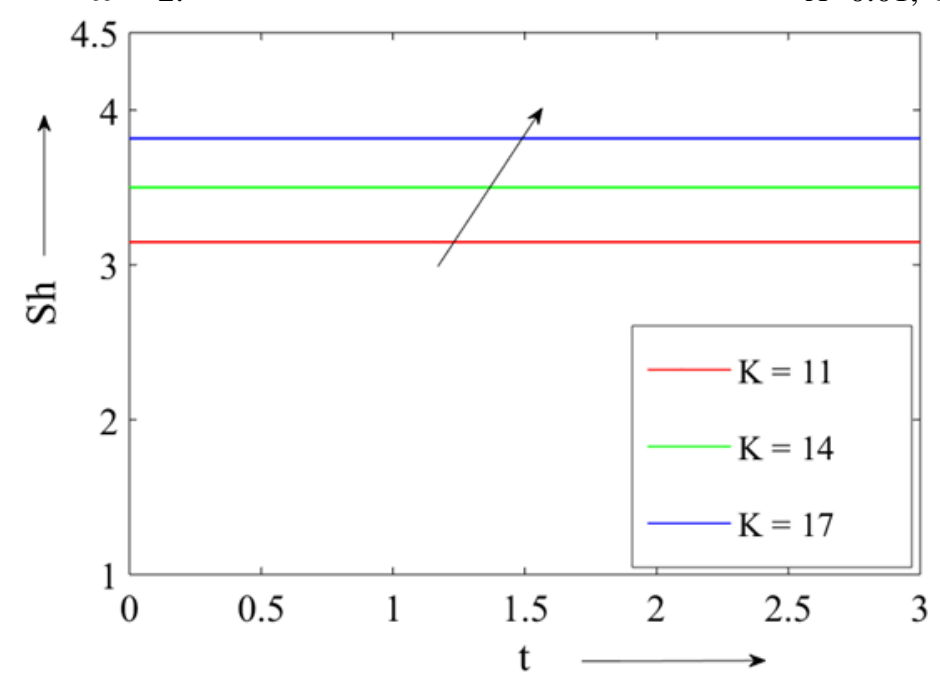

Figure 14. Sherwood number $S h$ vs. $t$ for variations in $K$ when $S c=0.7, \varepsilon=0.01, A=0.01, \omega=2$.

Figure 12 illustrates that the rate of heat transfer $\mathrm{Nu}$ increases for the increasing effect of Dufour number $D u$ or diffusion-thermo effect. But from Figure 13, it is seen that the rate of heat transfer decreases for high radiation. Figure 14 present the variation of Sherwood number against time $t$ under chemical reaction parameter $(\mathrm{K})$. It is seen in figure 14 that the mass transfer rate is enhanced under the chemical reaction effect, which indicates the fact that the rate of mass transfer increases for the consumption of species.

\section{Conclusions}

We have investigated MHD free convection from a semi-infinite vertical porous plate with a diffusion-thermo effect. We presented the results to illustrate the flow characteristics for the velocity, temperature, and concentration and the effects of the physical parameters of the flow. Therefore, we conclude that fluid velocity increases under the diffusion-thermo effect and thermal buoyancy force but decreases for increasing values of Hartmann number. The temperature of the fluid rises due to the Dufour effect but drops substantially under the radiation parameter effect. A significant downfall in fluid concentration is observed due to increased chemical reaction parameters and Schmidt number. Viscous drag at the plate increases under the diffusion-thermo effect, but it decreases for high radiation. The rate of heat transfer is enhanced with an increase in the Dufour effect, while a gradual decrement in this physical 
quantity is noted for high radiation. The rate of mass transfer rises under the chemical reaction effect.

\section{Funding}

This research received no external funding.

\section{Acknowledgments}

The authors would like to thank the anonymous reviewers for their valuable comments and suggestions to improve the 'paper's quality.

\section{Conflicts of Interest}

The authors declare no conflict of interest.

\section{References}

1. Alfven, H. Discovery of Alfven waves. Nature 1942, 150, 405-406.

2. Cowling, T.G. Magnetohydrodynamics. Reports on Progress in Physics 1962, 25, 244-286, https://doi.org/10.1088/0034-4885/25/1/307.

3. Shercliff, J.A. A text book of magnetohydrodynamics. Pergamon Press, London, 1965.

4. Ferraro, V.C.A.; Plumpton, C. An introduction to Magneto-fluid mechanics. Clarendon Press, Oxford, 1966.

5. Crammer, K.R.; Pai, S.I. Magneto fluid Dynamics for engineers and applied physicist. McGraw-Hill Book Co., New York, 1973.

6. Raghunath, K.; Obulesu, M.; Sivaprasad, R. Heat and mass transfer on an unsteady MHD flow through porous medium between two porous vertical plates. AIP Conference Proceedings 2020, 2220, https://doi.org/10.1063/5.0001103.

7. Anwar, T.; Kumam, P.; Watthayu, W. An exact analysis of unsteady MHD free convection flow of some nanofluids with ramped wall velocity and ramped wall temperature accounting heat radiation and injection/consumption. Scientific Reports 2020, 10, https://doi.org/10.1038/s41598-020-74739-w.

8. Goud, B.S.; Mahendar, D.; Shekar, M.N.R. Thermal radioactive influence on MHD free convection flow across a porous medium in a vertical surface with temperature. AIP Conference Proceedings 2020, 2246, https://doi.org/10.1063/5.0014524.

9. Sheri, S.R.; Jayaprasad, S.; Mahendar, D. Soret and Dufour effects on MHD free convection flow past an impulsively moving vertical plate in the presence of inclined magnetic field. AIP Conference Proceedings 2020, 2246, https://doi.org/10.1063/5.0015575.

10. Omamoke, E.; Amos, E.; Bunonyo, K.W.: Radiation and heat source effects on MHD free convection flow over an inclined porous plate in the presence of viscous dissipation. American Journal of Applied Mathematics 2020, 8, 190-206, https://doi.org/10.11648/j.ajam.20200804.14.

11. Seth, G.S.; Mahto, N.; Tripathi, R.; Bhattacharyya, A. Unsteady hydromagnetic flow formation with hall effect due to time-dependent free stream in a rotating medium, Journal of Nature Science and Sustainable Technology 2017, 11, 197-211..

12. Ahmed, N.; Choudhury, K. Heat and mass transfer in three-dimensional flow through a porous medium with periodic permeability. Heat Transfer-Asian Research 2019, 48, 644-662, https://doi.org/10.1002/htj.21399.

13. Sinha, A.; Ahmed, N. Hydromagnetic natural convection from a semi-infinite vertical porous plate in the presence of thermal radiation. JP Journal of Heat and Mass Transfer 2019, 17, 77-95, https://dx.doi.org/10.17654/HM017010077.

14. Allan, M.M.; Dardery, S.M. On the effect of convective heat and mass transfer on unsteady mixed convection MHD flow through vertical porous medium. International Journal of Latest Research in Engineering and Technology 2018, 13, 66-78, https://doi.org/10.5897/IJPS2017.4678.

15. Chamuah, K.; Ahmed, N.; Choudhury, K.: Oscillatory flow of a viscous conducting fluid through a uniformly moving vertical circular cylinder under pressure gradient. J. Math. Comput. Sci. 2021, 11, 4183-4202, https://doi.org/10.28919/jmcs/5806.

16. Ahmed, N.; Dutta, M. Natural convection in transient MHD dissipative flow past a suddenly started infinite vertical porous plate: a finite difference approach. Heat Transfer Research 2018, 49, 491-508, https://doi.org/10.1615/HeatTransRes.2018016823.

17. Mahato, R.; Das, M.; Sibanda, P. Hall effect on MHD transient free convection flow of chemically reactive Casson fluid with heat source/sink past an infinite vertical cylinder. Physica Scripta 2020, 96, https://doi.org/10.1088/1402-4896/abc5eb. 
18. Dagana, J.; Amos, E. MHD free convection heat and mass transfer flow in a porous medium with Dufour and chemical reaction effects. International Journal of Innovative Scientific \& Engineering Technologies Research 2020, 8, 1-12.

19. Reddy, B.S. Diffusion-thermo effects on heat and mass transfer of non-Newtonian power law fluid past a porous flat plate with chemical reaction. Int. J. Math. And Appl. 2018, 6, 1077-1083.

20. Ahmed, N.; Choudhury, K.; Chamuah, K.: Three dimensional hydromagnetic convective flow past a porous vertical plate with sinusoidal suction in slip flow regime. Mathematics in Engineering, Science and Aerospace. 2020, 11, 805-825.

21. Jha, B.K.; Sarki, M.N. Chemical reaction and Dufour effects on nonlinear free convection heat and mass transfer flow near a vertical moving porous plate. Heat Transfer 2020, 49, 984-999, https://doi.org/10.1002/htj.21649.

22. Islam, S.H.; Begum, P.; Sarma, D. Dufour Effect on MHD Free Convection Heat and Mass Transfer Effects Flow over an Inclined Plate Embedded in a Porous Medium. Journal of Scientific Research 2021, 13, 111123, http://dx.doi.org/10.3329/jsr.v13i1.48174.

23. Mishra, P.; Tripathi, S.: Effect of non-uniform heat source and radiation on unsteady MHD free convection flow past an infinite heated vertical plate in porous medium. E-Jurnal Matematika 2020, 9, 219-228, https://doi.org/10.24843/mtk.2020.v09.i04.p302.

24. Chiranjeevi, B.; Valsamy, P.; Vidyasagar, G. Radiation absorption on MHD free convective laminar flow over a moving vertical porous plate, viscous dissipation and chemical reaction with suction under the influence of transverses magnetic field. Materials Today: Proceedings 2021, 42, 1559-1569, https://doi.org/10.1016/j.matpr.2020.01.488.

25. Suneetha, K.; Ibrahim, S.; Reddy, G.; Kumar, P. Variable temperature and concentration impacts on radiative chemically magneto hydrodynamic viscoelastic fluid flow through porous moving plate. Mathematical Modelling of Engineering Problems, 2020, 7, 187-195, https://doi.org/10.18280/mmep.070203.

26. Goud, B.S. Thermal radiation influences on MHD stagnation point stream over a stretching sheet with slip boundary conditions. International Journal of Thermofluid Science and Technology 2020, 7, https://doi.org/10.36963/IJTST.2020070201.

27. Sharma, K.; Gupta, S. Radiation effects on MHD boundary layer flow and heat transfer along a stretching cylinder with variable thermal conductivity in a porous medium. Journal of Porous Media 2018, 21, 763779, https://doi.org/10.1615/JPorMedia.2018019284.

28. Daniel, Y.S.; Aziz, Z.A.; Ismail, Z.; Salah, F. Thermal radiation on unsteady electrical MHD flow of nanofluid over stretching sheet with chemical reaction. Journal of King Saud University - Science 2019, 31, 804-812, https://doi.org/10.1016/j.jksus.2017.10.002.

29. Prabhakar Ready, D.; Peter, J. Effects of Chemical Reaction on MHD Flow Past an Impulsively Started Infinite Vertical Plate with Variable Temperature and Mass Diffusion in the Presence of Hall Current. Journal of the Serbian Society for Computational Mechanics 2019, 13, 92-108, https://doi.org/10.3329/jname.v16i2.29526.

30. Suneetha, K.; Ibrahim, S.M.; Reddy, G.V.R. The effects of radiation and chemical reaction on MHD mixed convective flow and mass transfer from a vertical surface with Ohmic heating and viscous dissipation. Multidiscipline Modeling in Materials and Structures 2020, 16, 191-207, https://doi.org/10.1108/MMMS02-2018-0021.

31. Rajakumar, K.V.B.; Balamurugan, K.S.; Reddy, M.U.; Murthy, C.V.R. Radiation, dissipation and Dufour effects on MHD free convection Casson fluid flow through a vertical oscillatory porous plate with ion-slip current. International Journal of Heat and Technology 2018, 36, 494-508, https://doi.org/10.18280/ijht.360214.

32. Obulesu, M.; Raghunath, K.; Sivaprasad, R. Hall current effects on MHD convective flow past a porous plate with thermal radiation, chemical reaction with radiation absorption. AIP Conference Proceedings 2020, 2246, https://doi.org/10.1063/5.0014423.

33. Ahmed, N.; Bordoloi, R. Three-dimensional flow past a porous vertical plate in a porous medium with sinusoidal suction and permeability in the presence of thermal diffusion. Heat Transfer 2021, 1-24, https://doi.org/10.1002/htj.22325.

34. Seth, G.S.; Kumar, R.; Tripathi, R.; Bhattacharyya, A. Double diffusive MHD Casson fluid flow in a nonDarcy porous medium with Newtonian heating and thermo-diffusion effects. International Journal of Heat and Technology 2018, 36, 1517-1527, https://doi.org/10.18280/ijht.360446.

35. Kumar, R.; Bhattacharyya, A.; Seth, G.S.; Chamkha, A.J. Transportation of magnetite nanofluid flow and heat transfer over a rotating porous disk with Arrhenius activation energy: Fourth order Noumerov's method. Chinese Journal of Physics 2021, 69, 172-185, https://doi.org/10.1016/j.cjph.2020.11.018.

36. Cogley, A.C.L.; Vincenti, W.G.; Gilles, E.S. Differential approximation for radiative heat transfer in a nongrey gas near equilibrium. American Institute of Aeronautics and Astronautics 1968, 6, 551-553, https://doi.org/10.2514/3.4538. 